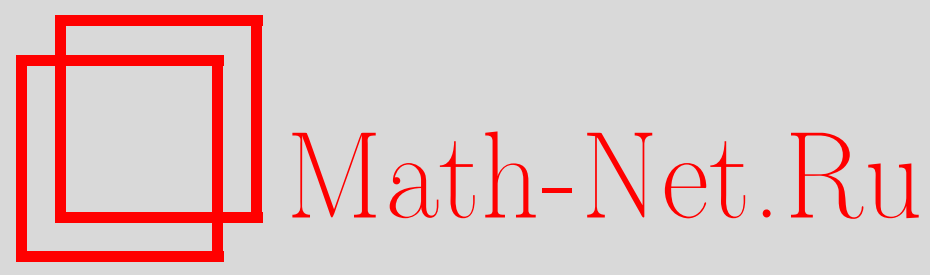

М. В. Фейгин, Квазиинварианты диэдральных систем, Maтем. заметки, 2004, том 76, выпуск 5, 776-791

DOI: https://doi.org/10.4213/mzm142

Использование Общероссийского математического портала Math-Net.Ru подразумевает, что вы прочитали и согласны с пользовательским соглашением http://www.mathnet.ru/rus/agreement

Параметры загрузки:

IP : 54.198 .187 .58

26 апреля 2023 г., 13:19:42

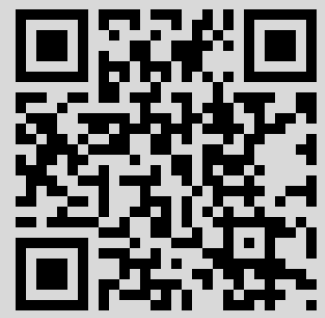


УДК 512

\title{
КВАЗИИНВАРИАНТЫ ДИЭДРАЛЬНЫХ СИСТЕМ
}

\author{
М. В. Фейгин
}

Для двумерных систем Кокстера с произвольными кратностями явно построен базис модуля квазиинвариантов над инвариантами. Доказано, что построенный базис состоит из $m$-гармонических многочленов, тем самым обобщены более ранние результаты Веселова и автора для систем с постоянной кратностью.

Библиографория: 15 названий.

1. Введение. Введем главньй объект наших исследований - алгебру квазиинвариантов. Рассмотрим систему Кокстера $\mathcal{R}$ в $\mathbb{R}^{n}$, состоящую из неколлинеарных пар векторов $\{ \pm \alpha\}$ с предписанными кратностями $m_{\alpha} \in \mathbb{Z}_{+}$. Соответствующая группа Кокстера $G$ порождена отражениями $s_{\alpha}, \alpha \in \mathcal{R}$ :

$$
s_{\alpha} u=u-\frac{2(\alpha, u)}{(\alpha, \alpha)} \alpha, \quad u \in \mathbb{R}^{n} .
$$

Группа $G$ должна быть конечна, и набор отражений $\left\{s_{\alpha}\right\}$ является набором всех отражений входящих в $G$. Функция кратности $m_{\alpha}=m(\alpha)$ предполагается инвариантной: $m_{\alpha}=m_{g(\alpha)}$ для любых $g \in G, \alpha \in \mathcal{R}$.

Полином $p(x)$ называется квазиинвариантом, связанным с системой $\mathcal{R}$, если он удовлетворяет условиям

$$
s_{\alpha} p(x)-p(x)=O\left((\alpha, x)^{2 m_{\alpha}+1}\right)
$$

вблизи гиперплоскости $(\alpha, x)=0$ для любого $\alpha \in \mathcal{R}$. Как легко видеть, множество квазиинвариантов образует кольцо. Эквивалентно (1) $p(x)$ является квазиинвариантом, если

$$
\partial_{\alpha}^{2 s-1} p=0 \quad \text { при }(\alpha, x)=0, \quad s=1, \ldots, m_{\alpha}, \quad \alpha \in \mathcal{R},
$$

где $\partial_{\alpha}-$ производная вдоль направления $\alpha$. Например, если полином $p(x)$ принадлежит кольцу $S^{G}$ полиномов, инвариантных относительно геометрического действия групшы Кокстера, то $s_{\alpha} p(x)=p(x)$ для любого $\alpha \in \mathcal{R}$; тем самым, свойство (1) вьполняется. Таким образом, кольцо квазиинвариантов $Q^{\mathcal{R}}$ содержит кольцо инвариантов $S^{G}$.

Кольца квазиинвариантов были введены Веселовьм и Чалых в 1990 году в контексте квантовых интегрируемых систем [1]. Авторы показали, что каждому квазиинварианту $q(x)$ соответствует дифференциальный оператор $\mathcal{L}_{q}=\chi(q)$ вида

$$
\mathcal{L}_{q}\left(x, \partial_{x}\right)=q\left(\partial_{x}\right)+\text { члены младших порядков по } \partial,
$$

На заключительном этапе работа вьполнена при финансовой поддержке Президента Р $\Phi$, грант № MK-2050.2003.01, и программы “Ведущие научные школы”, грант № НШ-2185.2003.1. 
и все такие операторы $\mathcal{L}_{q}$ коммутируют. При гомоморфизме $\chi$ квазиинвариант $q(x)=$ $x_{1}^{2}+\cdots+x_{n}^{2}$ переходит в обобщенный оператор Калоджеро-Мозера [2]-[4]

$$
\mathcal{L}_{x^{2}}=\Delta-\sum_{\alpha \in \mathcal{R}_{+}} \frac{2 m_{\alpha}}{(\alpha, x)} \partial_{\alpha}
$$

Здесь $\mathcal{R}_{+}$обозначает подмножество векторов из $\mathcal{R}$ принадлежащих некоторому полупространству, а $\Delta$ является оператором Лапласа в $\mathbb{R}^{n}$. Тем самьм, каждому квазиинварианту $q(x)$ отвечает квантовьй интеграл $\mathcal{L}_{q}$ задачи Калоджеро-Мозера (2). Как объясняется в [5], коммутативное кольцо всех квантовых интегралов для (2) изоморфно кольцу квазиинвариантов $Q^{\mathcal{R}}$.

Отметим, что на инвариантных полиномах гомоморфизм $\chi$ рассматривался Хекманом и Опдамом без условия целочисленности функции кратности [6]. Существенно, что для целочисленной функции кратности кольцо $Q^{\mathcal{R}}$ квантовых интегралов становится больше кольца инвариантов $S^{G}$, и кольцо интегралов зависит от выбранных целочисленных кратностей, как это было обнаружено Веселовым и Чалых [1]. Необходимость рассмотрения целых кратностей для получения бо́льших коммутативных колец была недавно доказана Танигучи [7].

Первое исследование кольца $Q^{\mathcal{R}}$ было проведено Волченко, Козачко и Мишачевым [8] в случае диэдральных систем с единичной кратностью. В работе [8] были найдены мультипликативные образующие кольца. Более полное описание кольца $Q^{\mathcal{R}}$ для диэдральных систем с постоянной кратностью $m_{\alpha}=m \in \mathbb{Z}_{+}$было сделано Веселовым и автором [5]. Оказалось, что для диэдральной системы $I_{2}(N)$, состоящей из $N$ прямых на плоскости $\mathbb{R}^{2} \cong \mathbb{C}$, кольцо $Q^{I_{2}(N)}$ является свободньм модулем над подкольцом инвариантов $S^{I_{2}(N)}=\mathbb{C}\left[z \bar{z}, z^{N}+\bar{z}^{N}\right]$. Этот модуль имеет $2 N$ образующих $h_{1}, \ldots, h_{2 N}$ таких, что линейное пространство $H_{m}=\left\langle h_{1} \ldots, h_{2 N}\right\rangle$, порожденное этими образующими, является пространством решений следующей системы из двух уравнений:

$$
\mathcal{L}_{z \bar{z}} h=0, \quad \mathcal{L}_{z^{N}+\bar{z}^{N}} h=0
$$

Также были найдены детерминантные формулы для $h_{i}$. В [9] было предположено, что кольцо квазиинвариантов $Q^{\mathcal{R}}$ является свободным модулем над инвариантами $S^{G}$ для любой кокстеровской системы $\mathcal{R}$. Также было предположено, что можно взять $m$-гармонические многочлены в качестве базиса этого свободного модуля. Напомним, что пространство $m$-гармонических многочленов $H_{m}$ состоит из решений следующей системы уравнений:

$$
\chi(\sigma) h=0
$$

где $\sigma$ является произвольным элементом из кольца $S_{+}^{G}$, инвариантных полиномов, не имеющих свободного члена (см. [9]).

Этингоф и Гинзбург доказали в [10], что кольцо $Q^{\mathcal{R}}$ действительно свободно над инвариантами $S^{G}$ для любой системы Кокстера $\mathcal{R}$. Однако выбор базиса в $H_{m}$ в качестве свободного базиса для квазиинвариантного модуля в общем случае невозможен (см. контрпример в [10]). Тем не менее, степени однородных образующих совпадают со степенями однородного базиса в $H_{m}$, как было доказано в [10]. Свободные образующие должны быть базисом в дополнении к идеалу, порожденному однородньми инвариантами положительной степени в $Q^{\mathcal{R}}$. Задача выбора такого базиса остается открытой. 
Помимо свойства коэн-маколевости для квазиинвариантной алгебры, Этингоф и Гинзбург также установили свойство горенштейновости [10]. Одно из двух доказательств, приведенное в [10], основано на результатах работы Фельдера и Веселова [11]. В последней работе, в частности, был найден полином Пуанкаре для пространства $m$-гармонических многочленов $H_{m}$. Свойство горенштейновости отвечает палиндромичности полинома Пуанкаре для $H_{m}$, предположенной в [9] и доказанной в [11].

Дифференциальные операторы на особых алгебраических многообразиях, соответствующих $Q^{\mathcal{R}}$, изучались Берестом, Этингофом и Гинзбургом. В работе [12] было доказано, что алгебра дифференциальньх операторов проста и Морита эквивалентна алгебре Вейля полиномиальных дифференциальных операторов в $n$-мерном пространстве.

В данной работе мы, следуя [13], описьваем квазиинварианты диэдральных систем $I_{2}(2 N)$ с произвольными (инвариантньми) кратностями $(m, n)^{1}$. Мы предлагаем явные формулы для образуюших кольца $Q^{I_{2}(2 N)}$ как модуля над инвариантами $S^{I_{2}(2 N)}$, обобщая формулы из [5]. Оказывается, что в этом случае, как и в случае диэдральных систем с постоянной кратностью [5], можно взять в качестве образующих однородньй базис пространства $m$-гармонических многочленов $H_{m}{ }^{2}$. Тем самьг, получаются явные детерминантные формулы для базисных $m$-гармонических многочленов для произвольных диэдральных систем.

Техника, используемая в данной работе, отличается от подхода в [5], где рассматривались диэдральные системы с постоянной кратностью. Теперь мы существенно используем результаты Этингофа и Гинзбурга [10], а также Фельдера и Веселова [11]. Такой подход позволяет заметно сократить явные вычисления. Схема рассмотрений такова.

В п. 2 напоминается результат Фельдера и Веселова о полиноме Пуанкаре для $m$-гармонических многочленов и явно выписьвается полином Пуанкаре $P_{H_{m}}$ для диэдральных $m$-гармоник. По теореме Этингофа и Гинзбурга степени однородных образующих для квазиинвариантов задаются $P_{H_{m}}$. В п. 3 доказьвается, что эти образуюшие могут быть взяты в определенной форме. Чтобы доказать это, мы рассуждаем следующим образом. Возьмем произвольный полином рассматриваемой степени и наложим условия квазиинвариантности. Из теоремы Этингофа и Гинзбурга следует, что эта система уравнений разрешима. Из вида уравнений следует, что она также должна иметь решение в нужной нам форме. Далее проверяется, что линейная оболочка выбранных квазиинвариантов не принадлежит идеалу, порожденному однородньми инвариантами положительной степени. Эту проверку можно легко сделать, вновь используя теорему Этингофа и Гинзбурга. Таким образом, мы заключаем (по теореме Этингофа-Гинзбурга), что построен свободньй базис с элементами определенного вида.

В п. 4 вначале проверяется, что построенный базис является $m$-гармоническим. Это следует из специального вида элементов, входящих в базис; здесь опять используется теорема Этингофа-Гинзбурга. Затем мы возвращаемся к системе квазиинвариантных условий, задающих наш базис. Мы замечаем, что для многочлена рассматриваемого вида набор квазиинвариантных условий приводит к $m$-гармоничности этого многочлена. В частности, полином рассматриваемого вида однозначно определяется набором ква-

\footnotetext{
${ }^{1}$ В этом случае имеется две орбиты относительно действия группы Кокстера $G$ на множестве корней $\mathcal{R}$. Кратность на одной из них равняется $m \in \mathbb{Z}_{+}$, и кратность равняется $n \in \mathbb{Z}_{+}$на второй орбите.

${ }^{2} \mathrm{~B}$ обозначении $H_{m}$, под $m$ имеется в виду функция кратности $m=m(\alpha)$. Для системы $I_{2}(2 N)$ функция кратности принимает два значения $m$ и $n$.
} 
зиинвариантных условий. Этот факт позволяет получить явные детерминантные формулы для $m$-гармонических многочленов и, тем самым, для базиса квазиинвариантного модуля.

2. Полином Пуанкаре для диэдральных систем. Пусть $P_{H_{m}}(t)$ обозначает полином Пуанкаре пространства $m$-гармонических многочленов $H_{m}$, связанных с системой Кокстера $\mathcal{R}$,

$$
P_{H_{m}}(t)=\sum_{i \geqslant 0} \operatorname{dim}\left(H_{m}^{(i)}\right) t^{i}
$$

где $H_{m}^{(i)}$ - пространство однородных $m$-гармонических полиномов степени $i$. Следующая формула для $P_{H_{m}}(t)$ была получена Фельдером и Веселовым в [11]:

$$
P_{H_{m}}(t)=\sum_{V_{j}} t^{\sum_{a} m_{a} d_{a}^{-}\left(V_{j}\right)} P_{j}(t)
$$

В этой формуле внешнее суммирование ведется по всем неизоморфным неприводимым представлениям групшы Кокстера $G$. Внутреннее суммирование ведется по классам $C_{a}$ сопряженных отражений в $G$. Полином $P_{j}$ обозначает полином Пуанкаре представления $V_{j}$ в пространстве $H_{0} \cong \mathbb{C}\left[x_{1}, \ldots, x_{n}\right] / I_{0}$, где $I_{0}$ - идеал в кольце полиномов, порожденньй однородньми инвариантами положительной степени. То есть коэффициент при $t^{k}$ равняется размерности изотипической компоненты представления $V_{j}$ в $k$-й градуировке $\mathbb{C}\left[x_{1}, \ldots, x_{n}\right] / I_{0}$. Далее,

$$
d_{a}^{-}\left(V_{j}\right)=\frac{2 N_{a} \operatorname{dim} V_{j, \alpha}^{-}}{\operatorname{dim} V_{j}}
$$

где $N_{a}$ - число элементов в классе $C_{a}$ и

$$
V_{j, \alpha}^{-}=\left\{v \in V_{j} \mid s_{\alpha} v=-v\right\}, \quad s_{\alpha} \in C_{a}
$$

$m_{a}$ - кратность отражений $s_{\alpha} \in C_{a}$.

Для диэдральной группы $I_{2}(N)$ с нечетным $N$ имеется два неизоморфных одномерных неприводимых представления и $(N-1) / 2$ двумерных. Все отражения сопряжены и $N_{a}=N$. Так как в комплексных координатах кольцо инвариантов изоморфно $\mathbb{C}\left[z \bar{z}, z^{N}+\bar{z}^{N}\right]$, можно выбрать образуюшие в факторпространстве следуюшим образом:

$$
\mathbb{C}[z, \bar{z}] / I_{0} \simeq\left\langle 1, z, \bar{z}, \ldots, z^{N-1}, \bar{z}^{N-1}, z^{N}-\bar{z}^{N}\right\rangle .
$$

Для одномерньг представлений часть суммы (3) имеет вид

$$
1+t^{(2 m+1) N}
$$

где первый член соответствует тривиальному представлению $V_{\text {triv }}$, реализованному на константах. Второй член соответствует знаковому представлению $V_{\mathrm{sign}}$, которое реализуется на векторе $z^{N}-\bar{z}^{N}$. Мы также пользуемся тем, что

$$
d_{a}^{-}\left(V_{\text {triv }}\right)=\frac{2 N \cdot 0}{1}=0, \quad d_{a}^{-}\left(V_{\mathrm{sign}}\right)=\frac{2 N \cdot 1}{1}=2 N .
$$


Теперь рассмотрим двумерные представления. Представление $V_{i}$ реализуется как $\left\langle z^{i}, \bar{z}^{i}\right\rangle$ и как $\left\langle z^{N-i}, \bar{z}^{N-i}\right\rangle$. Далее,

$$
d_{a}^{-}\left(V_{i}\right)=\frac{2 N \cdot 1}{2}=N
$$

Объединяя вычисления, получаем

$$
P_{H_{m}}=1+t^{(2 m+1) N}+\sum_{i=1}^{(N-1) / 2} 2 t^{m N}\left(t^{i}+t^{N-i}\right)=1+2 \sum_{i=1}^{N-1} t^{m N+i}+t^{(2 m+1) N}
$$

Теперь рассмотрим четные диэдральные системы $I_{2}(2 N)$. В группе имеется два класса $C_{a}$ и $C_{b}$ сопряженных отражений, каждьй из которых состоит из $N$ элементов. Обозначим кратности отражений через $m_{a}=m$ и $m_{b}=n$. Как и в нечетном случае,

$$
\mathbb{C}[z, \bar{z}] / I_{0} \simeq\left\langle 1, z, \bar{z}, \ldots, z^{2 N-1}, \bar{z}^{2 N-1}, z^{2 N}-\bar{z}^{2 N}\right\rangle .
$$

Вначале проанализируем одномерные представления и вычислим соответствующий кусок полинома Пуанкаре. Имеется четыре одномерных представления для $I_{2}(2 N)$, зависящих от того, действуют ли классы отражений $C_{a}, C_{b}$ как 1 или -1 .

- $V_{\text {triv }}=\langle 1\rangle$. Соответствуюший член в (3) равняется 1.

- $V_{\text {sign }}=\left\langle z^{2 N}-\bar{z}^{2 N}\right\rangle, d_{a}^{-}\left(V_{\text {sign }}\right)=d_{b}^{-}\left(V_{\text {sign }}\right)=\frac{2 N \cdot 1}{1}=2 N$. Вклад в полином Пуанкаре равен $t^{(m+n+1) 2 N}$.

- $V_{\text {sign }^{1}}=\left\langle z^{N}+\bar{z}^{N}\right\rangle$. Получаем

$$
m_{a} d_{a}^{-}\left(V_{\text {sign }^{1}}\right)+m_{b} d_{b}^{-}\left(V_{\text {sign }^{1}}\right)=m \cdot \frac{2 N \cdot 0}{1}+n \cdot \frac{2 N \cdot 1}{1}=2 n N,
$$

что приводит к члену $t^{(2 n+1) N}$ в $(3)$.

- $V_{\text {sign }^{2}}=\left\langle z^{N}-\bar{z}^{N}\right\rangle$. Получаем

$$
m_{a} d_{a}^{-}\left(V_{\mathrm{sign}^{2}}\right)+m_{b} d_{b}^{-}\left(V_{\mathrm{sign}^{2}}\right)=m \cdot \frac{2 N \cdot 1}{1}+n \cdot \frac{2 N \cdot 0}{1}=2 m N,
$$

что приводит к члену $t^{(2 m+1) N}$ в $(3)$.

Действительно, представления $V_{\text {sign }^{1}}, V_{\text {sign }^{2}}$ определяются свойством, что для любых $s \in C_{a}, \tau \in C_{b}$

$$
\left.s\right|_{V_{\mathrm{sign} 1}}=\mathrm{Id},\left.\quad s\right|_{V_{\mathrm{sign}^{2}}}=-\mathrm{Id},\left.\quad \tau\right|_{V_{\mathrm{sign}^{1}}}=-\mathrm{Id},\left.\quad \tau\right|_{V_{\mathrm{sign}^{2}}}=\mathrm{Id}
$$

Группа диэдра порождается двумя отражениями $s \in C_{a}, \tau \in C_{b}$ такими, что $(s \tau)^{2 N}=1$. Она также порождается $s$ и $s \tau$. В наших обозначениях мы можем думать об $s$ как об отражении относительно прямой $z=\bar{z}$, и $s \tau$ является поворотом на угол $\phi=\pi / N$. Таким образом, верны следующие формулы:

$$
s: z \rightarrow \bar{z}, \quad \bar{z} \rightarrow z, \quad s \tau: z \rightarrow \varepsilon z, \quad \bar{z} \rightarrow \bar{\varepsilon} \bar{z},
$$


где $\varepsilon=e^{i \pi / N}$. Далее, представление $V_{\operatorname{sign}^{1}}$ характеризуется свойством

$$
\left.s\right|_{V_{\text {sign } 1}}=\mathrm{Id},\left.\quad \tau\right|_{V_{\text {sign } 1}}=-\mathrm{Id}
$$

или, эквивалентно,

$$
\left.s\right|_{V_{\text {sign }^{1}}}=\mathrm{Id},\left.\quad(s \tau)\right|_{V_{\text {sign } 1}}=-\mathrm{Id} .
$$

Очевидно, групшовые элементы действуют следуюшим образом:

$$
s\left(z^{N}+\bar{z}^{N}\right)=\bar{z}^{N}+z^{N}
$$

и

$$
(s \tau)\left(z^{N}+\bar{z}^{N}\right)=\varepsilon^{N} z^{N}+\bar{\varepsilon}^{N} \bar{z}^{N}=-\left(z^{N}+\bar{z}^{N}\right) .
$$

Таким образом, $V_{\text {sign }^{1}}=\left\langle z^{N}+\bar{z}^{N}\right\rangle$, и если $m_{s}=m, m_{\tau}=n$, то вклад данного неприводимого представления в полином Пуанкаре будет $t^{(2 n+1) N}$. Аналогично, $V_{\mathrm{sign}^{2}}=$ $\left\langle z^{N}-\bar{z}^{N}\right\rangle$, так как

$$
s\left(z^{N}-\bar{z}^{N}\right)=\bar{z}^{N}-z^{N}=-\left(z^{N}-\bar{z}^{N}\right)
$$

и

$$
(s \tau)\left(z^{N}-\bar{z}^{N}\right)=\varepsilon^{N} z^{N}-\bar{\varepsilon}^{N} \bar{z}^{N}=-\left(z^{N}-\bar{z}^{N}\right)
$$

соответствующий член в полиноме Пуанкаре равен $t^{(2 m+1) N}$.

Теперь обратимся к двумерньм неприводимым представлениям. Представление $V_{i}$ реализуется дважды в $\mathbb{C}[z, \bar{z}] / I_{0}$ как пространство $\left\langle z^{i}, \bar{z}^{i}\right\rangle$ и как $\left\langle z^{2 N-i}, \bar{z}^{2 N-i}\right\rangle, i=$ $1, \ldots, N-1$. Таким образом, $P_{i}(t)=2 t^{i}+2 t^{2 N-i}$ в (3). Для всех отражений $s \in C_{a}$, $\tau \in C_{b}$ вьполняется $\operatorname{dim} V_{i}^{-}=1$; следовательно,

$$
d_{a}^{-}\left(V_{i}\right)=d_{b}^{-}\left(V_{i}\right)=\frac{2 N \cdot 1}{2}=N
$$

и мы получаем член полинома Пуанкаре, равный $2 t^{(m+n) N}\left(t^{i}+t^{2 N-i}\right)$. Суммируя, получаем

$$
P_{H_{m}}=1+t^{(m+n+1) 2 N}+t^{(2 m+1) N}+t^{(2 n+1) N}+2 \sum_{i=1}^{N-1} t^{(m+n) N}\left(t^{i}+t^{2 N-i}\right) .
$$

3. Образующие квазиинвариантов. Напомним следующую ключевую теорему, доказанную Этингофом и Гинзбургом [10] (см. также [9]).

Tеорема 1 [10]. Для любой системы Кокстера $\mathcal{R}$ кольцо квазиинвариантов $Q^{\mathcal{R}}$ является свободным модулем над подкольиом инвариантов $S^{G}$ соответствующей группы Кокстера $G$. В качестве образующих можно взять базис однородных представителей факторпространства $Q^{\mathcal{R}} / I$, где $I-$ идеал в $Q^{\mathcal{R}}$, порожсднный однородными инвариантными полиномами полохительных степеней. Степени однородных образующих совпадают со степенями однородного базиса в пространстве т-гармонических многочленов $H_{m}$.

Мы собираемся определить, каковы полиномы, которые могут быть выбраны как образующие для диэдральных систем. Так как случай постоянной кратности уже был рассмотрен в работе [9], будем считать, что наша групша является четной диэдральной 
группой $I_{2}(2 N)$ с различньми кратностями, для определенности $m>n$. По теореме 1 степени образующих для $Q^{I_{2}(2 N)}$ над инвариантами совпадают со степенями базисных $m$-гармонических полиномов. В соответствии с (5) они удовлетворяют

$$
(2 n+1) N<(m+n) N+i<(2 m+1) N<(m+n+1) 2 N,
$$

где $1 \leqslant i \leqslant 2 N-1, i \neq N$. И мы получаем следующую таблицу для числа образующих в определенной степени.

ТАБЛИЦА 1

\begin{tabular}{|c|c|c|c|c|c|}
\hline степень & 0 & $(2 n+1) N$ & $(m+n) N+i$ & $(2 m+1) N$ & $(m+n+1) 2 N$ \\
\hline число образующих & 1 & 1 & 2 & 1 & 1 \\
\hline
\end{tabular}

Введем нормальные вектора $\alpha_{i}, i=0, \ldots, 2 N-1$, к линиям отражений. Именно, $\alpha_{i}=(-\sin (\pi i /(2 N)), \cos (\pi i /(2 N)))$, положим кратности $m_{i}=m$ для четных $i$ и $m_{i}=n$ для нечетных $i$. Введем следующие четыре квазиинварианта:

$$
\begin{gathered}
q^{0}=1, \quad q^{1}=\left(z^{N}+\bar{z}^{N}\right)^{2 n+1}=\mu_{1} \prod_{\substack{i=1 \\
i=2 j+1}}^{2 N-1}\left(\alpha_{i}, x\right)^{2 n+1}, \\
q^{2}=\left(z^{N}-\bar{z}^{N}\right)^{2 m+1}=\mu_{2} \prod_{\substack{i=0 \\
i=2 j}}^{2 N-2}\left(\alpha_{i}, x\right)^{2 m+1}, \\
q^{3}=\left(z^{N}+\bar{z}^{N}\right)^{2 n+1}\left(z^{N}-\bar{z}^{N}\right)^{2 m+1}=\mu_{3} \prod_{i=0}^{2 N-1}\left(\alpha_{i}, x\right)^{2 m_{i}+1},
\end{gathered}
$$

где $\mu_{1}, \mu_{2}, \mu_{3}$ - некоторые константы. Очевидно, что $q^{0}$ и $q^{3}$ являются квазиинвариантами. Полином $q^{1}$ такжеквазиинвариантен. Действительно, условия на прямых $\left(\alpha_{i}, x\right)=0$ с нечетным $i$, очевидно, вьполняются. Если $i$ четно, то условия следуют из инвариантности $s_{\alpha_{i}} q^{1}=q^{1}$. Аналогично, $q^{2} \in Q^{I_{2}(2 N)}$. Эти квазиинварианты будут составлять часть базиса для $Q^{I_{2}(2 N)}$ над инвариантами, который мы строим.

ПРЕДЛОЖЕНИЕ 1. Квазиинварианты $q^{0}, q^{1}, q^{2}, q^{3}$, определенные в (6), не принадлежат идеалу $I$, порожденному однородными инвариантами положительной степени $S_{+}^{I_{2}(2 N)}$ в кольие $Q^{I_{2}(2 N)}$.

ДокАЗАТЕЛЬСТВо. В соответствии с теоремой 1 степени свободных образующих для $Q^{I_{2}(2 N)}$ над инвариантами заданы таблищей 1 . Тем самым, не существует квазиинвариантов степени меньше, чем $\operatorname{deg} q^{1}$, которые не являются инвариантами. Так как $q^{1}$ не является инвариантом, мы заключаем, что $q^{1} \notin I$. Покажем, что $q^{2} \notin I$. Пусть $r_{i}^{1,2}$ - независимые элементы в дополнении к $I$ степеней $(m+n) N+i, 1 \leqslant i \leqslant 2 N-1$, $i \neq N$. Предположим, что $q_{2} \in I$, т.е.

$$
q^{2}=s_{0} q^{0}+s_{1} q^{1}+\sum_{i} s_{i}^{1} r_{i}^{1}+\sum_{i} s_{i}^{2} r_{i}^{2}
$$

где $s_{0}, s_{1}, s_{i}^{1}, s_{i}^{2}$ инварианты, т.е. элементы $\mathbb{C}\left[z \bar{z}, z^{2 N}+\bar{z}^{2 N}\right]$. Так как $\operatorname{deg} r_{i}^{1,2} \neq 0 \bmod N$, полиномы $s_{i}^{1,2}$ делятся на $z \bar{z}$. Рассмотрим соотношение (7) по модулю членов, деляшихся на $z \bar{z}$. Получаем

$$
z^{(2 m+1) N}-\bar{z}^{(2 m+1) N}=\lambda_{1}\left(z^{2 N}+\bar{z}^{2 N}\right)^{a}\left(z^{(2 n+1) N}+\bar{z}^{(2 n+1) N}\right)+\lambda_{2}\left(z^{2 N}+\bar{z}^{2 N}\right)^{b}+O(z \bar{z}) .
$$


Теперь мы приходим к противоречию, так как получаем одновременно $\lambda_{1}+\lambda_{2}=1$ и $\lambda_{1}+\lambda_{2}=-1$. Тем самым, соотношение (7) невозможно и $q^{2} \notin I$.

Покажем, наконец, что $q^{3} \notin I$. Как и вьше, предполагая обратное, получаем, что должно вьполняться следующее соотношение:

$$
\begin{aligned}
z^{2(m+n+1) N}-\bar{z}^{2(m+n+1) N}= & \lambda_{1}\left(z^{2 N}+\bar{z}^{2 N}\right)^{a}\left(z^{(2 m+1) N}-\bar{z}^{(2 m+1) N}\right) \\
& +\lambda_{2}\left(z^{2 N}+\bar{z}^{2 N}\right)^{b}\left(z^{(2 n+1) N}+\bar{z}^{(2 n+1) N}\right) \\
& +\lambda_{3}\left(z^{2 N}+\bar{z}^{2 N}\right)^{c}+O(z \bar{z})
\end{aligned}
$$

Отсюда $\lambda_{1}=\lambda_{2}=0$, так как для любых $a, b$ степени мономов по $z$ и по $\bar{z}$, получаемые из первых двух членов правой части (8), имеют вид $N$ • (нечетное число). А в левой части результат деления степени на $N$ равен $2(m+n+1)$, что четно. Далее, заключаем, что правая часть (8) принимает вид $\lambda_{3}\left(z^{2 N c}+\bar{z}^{2 N c}\right)+O(z \bar{z})$ и равенство (8) невозможно. Следовательно, $q^{3} \notin I$, и предложение доказано.

Доказанное предложение показывает, что полиномы $q^{0}, q^{1}, q^{2}, q^{3}$ могут быть выбраны как часть свободного базиса $Q^{I_{2}(2 N)}$ над инвариантами. Оставшиеся $2 N-4$ многочлена в базисе состоят из пар многочленов степеней $(m+n) N+i$ при $1 \leqslant i \leqslant 2 N-1$, $i \neq N$. Следуюшие предложение и теорема показьвают, что эти квазиинварианты могут быть выбраны в более или менее простой форме.

ПРЕДЛОЖЕНИЕ 2. Существуют квазиинварианты $q_{i}^{1,2}$ вида

$$
q_{i}^{1}=\sum_{s=0}^{m+n} a_{s} z^{(m+n-s) N+i} \bar{z}^{N s}, \quad a_{0}=1, \quad q_{i}^{2}=\sum_{s=0}^{m+n} b_{s} \bar{z}^{(m+n-s) N+i} z^{N s}, \quad b_{s}=\bar{a}_{s}
$$

для $1 \leqslant i \leqslant 2 N-1, i \neq N$.

ДокАЗАТЕЛЬСТво. Обозначим через $r_{i}^{1,2}$ какой-нибудь базис в однородном дополнении к $I$ в степени $(m+n) N+i, 1 \leqslant i \leqslant 2 N-1, i \neq N$. Рассмотрим двумерное пространство $\left\langle r_{i}^{1}, r_{i}^{2}\right\rangle$. Так как оно не пересекает $I$, мы можем считать, что

$$
r_{i}^{1}=z^{(m+n) N+i}+O(z \bar{z}), \quad r_{i}^{2}=\bar{z}^{(m+n) N+i}+O(z \bar{z}) .
$$

Рассмотрим квазиинвариантные условия для первого многочлена:

$$
r_{i}^{1}=\sum_{s=0}^{(m+n) N+i-1} a_{s} z^{(m+n) N+i-s} \bar{z}^{s}, \quad a_{0}=1
$$

Прямые $\left(\alpha_{j}, x\right)=0, j=0, \ldots, 2 N-1$, задаются уравнениями $z=\varepsilon^{j} \bar{z}$, где $\varepsilon^{2 N}=1$, и $\varepsilon-$ примитивньй корень. Условия квазиинвариантности $\partial_{\alpha_{j}}^{2 t-1} r_{i}^{1}=0$ при $\left(\alpha_{j}, x\right)=0 \Longleftrightarrow$ $z=\varepsilon^{j} \bar{z}$ эквивалентны уравнениям

$$
\sum_{s=0}^{(m+n) N+i-1} a_{s}((m+n) N+i-2 s)^{2 t-1} \varepsilon^{((m+n) N+i-s) j}=0
$$


или, эквивалентно,

$$
\sum_{s=0}^{(m+n) N+i-1} a_{s}((m+n) N+i-2 s)^{2 t-1}\left(\frac{1}{\varepsilon^{s}}\right)^{j}=0 .
$$

Пусть $t$ удовлетворяет $1 \leqslant t \leqslant n<m$. Тогда условия квазиинвариантности нетривиальны для всех $2 N$ прямых $j=0, \ldots, 2 N-1$. Эти условия можно переписать в виде

$$
\sum_{p=0}^{2 N-1} A_{p}\left(\frac{1}{\varepsilon^{p}}\right)^{j}=0
$$

где

$$
A_{p}=\sum_{s \equiv p(2 N)} a_{s}((m+n) N+i-2 s)^{2 t-1} .
$$

Последняя система уравнений имеет вид системы Вандермонда. Так как $\varepsilon^{p_{1}} \neq \varepsilon^{p_{2}}$, если $p_{1} \neq p_{2}$, мы заключаем, что

$$
A_{p}=0, \quad p=0, \ldots, 2 N-1 .
$$

Теперь рассмотрим оставшиеся квазиинвариантные условия, соответствующие $n<$ $t \leqslant m$. Эти условия соответствуют прямьм с четным номером $j$. Имеем

$$
\sum_{s=0}^{(m+n) N+i-1} a_{s}((m+n) N+i-2 s)^{2 t-1}\left(\frac{1}{\varepsilon^{s}}\right)^{j}=0,
$$

где $j=0,2, \ldots, 2 N-2$. Эквивалентно,

$$
\sum_{p=0}^{N-1} B_{p}\left(\frac{1}{\varepsilon^{2 p}}\right)^{j}=0
$$

где

$$
B_{p}=\sum_{s \equiv p(N)} a_{s}((m+n) N+i-2 s)^{2 t-1}
$$

и $j=0,1, \ldots, N-1$. Эта система уравнений также имеет вид системы Вандермонда. Так как $\varepsilon^{2 p_{1}} \neq \varepsilon^{2 p_{2}}$, если $p_{1} \neq p_{2}$ и $0 \leqslant p_{i} \leqslant N-1$, заключаем, что

$$
B_{p}=0, \quad p=0, \ldots, N-1 .
$$

Таким образом, квазиинвариантность полинома

$$
r_{i}^{1}=\sum_{s=0}^{(m+n) N+i-1} a_{s} z^{(m+n) N+i-s} \bar{z}^{s}
$$

эквивалентна следуюшим условиям на его коэффициенты:

$$
\begin{aligned}
& \sum_{s \equiv p(2 N)} a_{s}((m+n) N+i-2 s)^{2 t-1}=0, \quad p=0,1, \ldots, 2 N-1, \quad 1 \leqslant t \leqslant n, \\
& \sum_{s \equiv p(N)} a_{s}((m+n) N+i-2 s)^{2 t-1}=0, \quad p=0,1, \ldots, N-1, \quad n<t \leqslant m .
\end{aligned}
$$


Эти уравнения распадаются на системы уравнений на наборы коэффищиентов $\left\{a_{s}\right\}$ с индексами $s$, имеющими одинаковьй остаток после деления на $N$. Так как у системы (10) имеется решение с $a_{0}=1$, заключаем, что имеется и решение, в котором единственные ненулевые коэффициенты суть

$$
a_{0}, a_{N}, a_{2 N}, \ldots, a_{(m+n+\delta) N},
$$

где $\delta=0$, если $1 \leqslant i \leqslant N-1$, и $\delta=1$, если $N+1 \leqslant i \leqslant 2 N-1$. Соответствующий квазиинвариант имеет вид

$$
R_{i}^{1}=\sum_{s=0}^{m+n+\delta} a_{s N} z^{(m+n-s) N+i} \bar{z}^{N s}
$$

с коэффициентами, удовлетворяющими следующей системе уравнений:

$$
\begin{aligned}
& \sum_{\substack{0 \leqslant m+n+\delta \\
s=2 k}} a_{s N}((m+n-2 s) N+i)^{2 t-1}=0, \quad 1 \leqslant t \leqslant n \\
& \sum_{\substack{0 \leqslant s \leqslant m+n+\delta \\
s=2 k+1}} a_{s N}((m+n-2 s) N+i)^{2 t-1}=0, \quad 1 \leqslant t \leqslant n \\
& \sum_{s=0}^{m+n+\delta} a_{s N}((m+n-2 s) N+i)^{2 t-1}=0, \quad n<t \leqslant m .
\end{aligned}
$$

Так как уравнения квазиинвариантности (10) (или (12)) вещественны, комплексно-сопряженньй к квазиинварианту также является квазиинвариантом. Тем самьп, существуют квазиинварианты

$$
R_{i}^{2}=\bar{R}_{i}^{1}=\sum_{s=0}^{m+n+\delta} b_{s N} \bar{z}^{(m+n-s) N+i} z^{N s}
$$

с $b_{s N}=\bar{a}_{s N}$. Для $i \leqslant N-1$ мы имеем $\delta=0$ и можем определить $q_{i}^{1}=R_{i}^{1}$. Далее, $q_{i}^{1}$ имеют вид, требуемый в утверждении предложения. Для $i \geqslant N+1$ последний ненулевой коэффищиент в $R_{i}^{1}$ есть $a_{(m+n+1) N}$. Покажем, что можно сделать $a_{(m+n+1) N}=0$. Для этого рассмотрим квазиинвариант

$$
q_{i}^{1}=R_{i}^{1}-a_{(m+n+1) N}(z \bar{z})^{i-N} R_{2 N-i}^{2} .
$$

Имеем

$$
\begin{aligned}
(z \bar{z})^{i-N} R_{2 N-i}^{2} & =(z \bar{z})^{i-N} \sum_{s=0}^{m+n} b_{s N} \bar{z}^{(m+n-s) N+2 N-i} z^{N s} \\
& =\sum_{s=0}^{m+n} b_{s N} \bar{z}^{(m+n-s+1) N} z^{N s+i-N} \\
& =\sum_{\widetilde{s}=0}^{m+n} b_{(m+n-\widetilde{s}) N} z^{(m+n-\widetilde{s}) N+i-N} \bar{z}^{N \widetilde{s}+N} .
\end{aligned}
$$


Тем самьм,

$$
q_{i}^{1}=z^{(m+n) N+i}+\sum_{s=1}^{m+n+1} z^{(m+n-s) N+i} \bar{z}^{N s}\left(a_{s N}-a_{(m+n+1) N} b_{m+n-s+1}\right),
$$

и последний член в сумме выше равняется нулю, так как $b_{0}=1$. Таким образом, $q_{i}^{1}$ для $i \geqslant N+1$ также имеет вид, требуемьй в предложении. Применяя комплексное сопряжение, мы получаем квазиинварианты в виде

$$
q_{i}^{2}=\sum_{s=0}^{m+n} b_{s} \bar{z}^{(m+n-s) N+i} z^{N s}, \quad b_{s}=\bar{a}_{s} .
$$

Предложение доказано.

ТЕОрема 2. Квазиинварианты $q_{i}^{1,2}$ вместе $с q^{0}, q^{1}, q^{2}, q^{3}$ образуют свободный базис для $Q^{I_{2}(2 N)}$ над $S^{I_{2}(2 N)}$.

ДокАЗАТЕЛЬСтво. По теореме 1 множество векторов образует базис для $Q^{I_{2}(2 N)}$ над $S^{I_{2}(2 N)}$, если этот набор образует базис в дополнении к идеалу $I$. По предложению 1 полиномы $q^{0}, q^{1}, q^{2}, q^{3}$ образуют часть базиса для $Q^{I_{2}(2 N)}$ над инвариантами. Нам остается доказать, что построенные в предложении 2 квазиинварианты не принадлежат идеалу $I$, порожденному однородными инвариантами положительной степени. Более точно, нужно доказать, что линейная оболочка $\left\langle q_{i}^{1}, q_{i}^{2}\right\rangle \cap I=0$. Если пересечение нетривиально, то

$$
\lambda_{1} q_{i}^{1}+\lambda_{2} q_{i}^{2} \in I
$$

и

$$
\lambda_{1} z^{(m+n) N+i}+\lambda_{2} \bar{z}^{(m+n) N+i}+O(z \bar{z})=p(z, \bar{z}) \in I,
$$

где $O(z \bar{z})$ является полиномом, делящимся на $z \bar{z}$, а $\lambda_{1}, \lambda_{2}-$ некоторые константы. Теперь в правой части мы можем представить $p(z, \bar{z})$ как линейную комбинацию базисных векторов для дополнения к $I$, умноженных на полиномиальные коэффициенты по $z \bar{z}$ и $z^{2 N}+\bar{z}^{2 N}$. Степени базисных векторов приведены в таблище 1 . Мы видим, что не существует базисных векторов со степенями меньшими, чем $(m+n) N+i$, но равными $(m+n) N+i$ по модулю $2 N$. Тем самым, $p(z, \bar{z})$ должен делиться на $z \bar{z} ;$ следовательно,

$$
\lambda_{1} z^{(m+n) N+i}+\lambda_{2} \bar{z}^{(m+n) N+i} \quad \text { делится на } z \bar{z},
$$

что возможно, только если $\lambda_{1}=\lambda_{2}=0$. Таким образом, теорема доказана.

4. Явные формулы для $m$-гармонического базиса. Покажем, что базис для $Q^{I_{2}(2 N)}$, рассмотренньй в теореме 2 , является в действительности $m$-гармоническим. Обозначим квантовые интегралы, соответствующие инвариантам $z \bar{z}$ и $z^{2 N}+\bar{z}^{2 N}$, через $\mathcal{L}_{1}$ и $\mathcal{L}_{2}$ соответственно. Соответствующие операторы имеют вид (cp. [5])

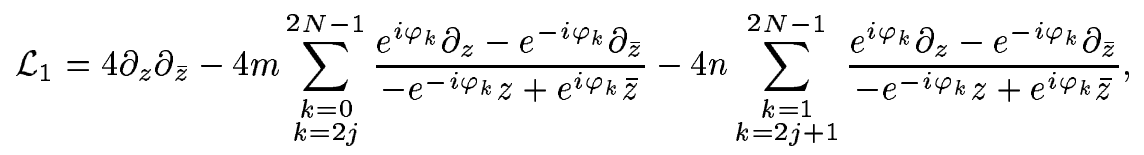

где $\varphi_{k}=\pi k /(2 N)$. Оператор $\mathcal{L}_{2}$ имеет следующий вид (с точностью до мультипликативной константы):

$$
\mathcal{L}_{2}=\partial_{z}^{2 N}+\partial_{\bar{z}}^{2 N}+\text { члены младших порядков по } \partial .
$$


ТЕОРемА 3. Полиномы (6), (9) являются $m$-гармоническими, т.е. выполняются следуюшие равенства:

1) $\mathcal{L}_{1}\left(q^{s}\right)=\mathcal{L}_{2}\left(q^{s}\right)=0,0 \leqslant s \leqslant 3$;

2) $\mathcal{L}_{1}\left(q_{i}^{s}\right)=\mathcal{L}_{2}\left(q_{i}^{s}\right)=0,1 \leqslant i \leqslant 2 N-1, i \neq N, s=1,2$.

ДокАЗАТЕЛЬСтво. Установим вначале, что полиномы $q^{s} m$-гармонические. Имеем $\mathcal{L}_{1}(1)=\mathcal{L}_{2}(1)=0$, так как $\operatorname{deg} \mathcal{L}_{i}(1)<0$, но, с другой стороны, $\mathcal{L}_{i}\left(Q^{I_{2}(2 N)}\right) \subset Q^{I_{2}(2 N)}$ (см. [9], где это объяснено в общем случае), т.е. образ полиномиален. Далее, докажем, что квазиинвариант $q^{3} m$-гармонический. Заметим, что

$$
q^{3}=\mu_{3} \prod_{i=0}^{2 N-1}\left(\alpha_{i}, x\right)^{2 m_{i}+1}
$$

характеризуется свойством, что он является антиинвариантным квазиинвариантом для группы $I_{2}(2 N)$ минимальной возможной степени. Так как операторы $\mathcal{L}_{1}, \mathcal{L}_{2}$ являются $I_{2}(2 N)$-инвариантными и так как их степени отрищательны, получаем

$$
\mathcal{L}_{1}\left(q^{3}\right)=\mathcal{L}_{2}\left(q^{3}\right)=0
$$

Квазиинварианты $q^{1}, q^{2}$ являются антиинвариантньми квазиинвариантами минимальной возможной степени для подгрупп $I_{2}(N) \subset I_{2}(2 N)$, соответствуюших двум разньп орбитам зеркал системы $I_{2}(2 N)$. Так как $\mathcal{L}_{1}, \mathcal{L}_{2}$ инвариантны по отношению к этим подгруппам, мы получаем оставшиеся равенства из первого утверждения теоремы, а именно,

$$
\mathcal{L}_{1}\left(q^{1,2}\right)=\mathcal{L}_{2}\left(q^{1,2}\right)=0 .
$$

Перейдем ко второй части. Рассмотрим двумерное пространство $V=\left\langle q_{i}^{1}, q_{i}^{2}\right\rangle$, порожденное полиномами $q_{i}^{1}, q_{i}^{2}$. Введем двумерные пространства $U, W$, порожденные образом $V$ при действии операторов $\mathcal{L}_{1}, \mathcal{L}_{2}$, а именно,

$$
U=\left\langle\mathcal{L}_{1} q_{i}^{1}, \mathcal{L}_{1} q_{i}^{2}\right\rangle, \quad W=\left\langle\mathcal{L}_{2} q_{i}^{1}, \mathcal{L}_{2} q_{i}^{2}\right\rangle
$$

Так как операторы $\mathcal{L}_{1}, \mathcal{L}_{2}$ инвариантны при действии $I_{2}(2 N)$, они являются сплетающими операторами между представлениями $V, U$ и между $V, W$ соответственно. По лемме Шура эти операторы либо нулевые, либо один или два из них являются скалярными операторами. Первьй случай соответствует условиям 2) в утверждении теоремы. Во втором случае соответствующее представление $U$ (или $W$ ) изоморфно представлению $V$.

Представление $V$ является двумерным неприводимым представлением. В базисе $q_{i}^{1}, q_{i}^{2}$ порождающие матрицы $s$ и $s \tau$ (см. (4)) принимают вид

$$
s=\left(\begin{array}{ll}
0 & 1 \\
1 & 0
\end{array}\right), \quad s \tau=(-1)^{m+n}\left(\begin{array}{cc}
\varepsilon^{i} & 0 \\
0 & \varepsilon^{-i}
\end{array}\right) .
$$

Для любого $q \in W$ имеем $\operatorname{deg} q=(m+n) N+i-2 N<(m+n) N$. Так как $Q^{I_{2}(2 N)}$ свободно порождается $q^{i}, q_{i}^{1,2}$ над $S^{I_{2}(2 N)}$, двумерные неприводимые представления в $Q^{I_{2}(2 N)}$ встречаются в степенях, больших или равных

$$
\min _{i}\left(\operatorname{deg} q_{i}^{1,2}\right)=(m+n) N+1 .
$$


Следовательно, $W=0$. Покажем, что также $U=0$. Заметим, что, как следует из (14), среди представлений $V_{i}=\left\langle q_{i}^{1}, q_{i}^{2}\right\rangle$ каждое неприводимое двумерное представление $I_{2}(2 N)$ встречается дважды. Именно, $V_{i} \cong V_{2 N-i}$, и при этом изоморфизме

$$
q_{i}^{1} \rightarrow \lambda q_{2 N-i}^{2}, \quad q_{i}^{2} \rightarrow \lambda q_{2 N-i}^{1}
$$

Если $1 \leqslant i \leqslant N-1$, то представление $V_{i}$ не встречается в пространстве $Q^{I_{2}(2 N)}$ в степенях, меньших $i$. Тем самым,

$$
\mathcal{L}_{1}\left(q_{i}^{1}\right)=\mathcal{L}_{1}\left(q_{i}^{2}\right)=0
$$

Нам остается рассмотреть случай $N+1 \leqslant i \leqslant 2 N-1$. Вообще говоря, мы имеем

$$
\mathcal{L}_{1}\left(q_{i}^{1}\right)=p q_{2 N-i}^{2}, \quad \mathcal{L}_{1}\left(q_{i}^{2}\right)=\bar{p} q_{2 N-i}^{1},
$$

где $p \in S^{I_{2}(2 N)}$. Покажем, что должно выполняться равенство $p=0$. Вспомним, что

$$
q_{i}^{1}=\sum_{s=0}^{m+n} a_{s} z^{(m+n-s) N+i} \bar{z}^{N s}, \quad q_{2 N-i}^{2}=\sum_{s=0}^{m+n} b_{s} \bar{z}^{(m+n-s) N+2 N-i} z^{N s} .
$$

Заметим, что степень $q_{i}^{1}$ по отношению к переменной $\bar{z}$ равняется $(m+n) N$. Ввиду формы (13) оператора $\mathcal{L}_{1}$ получаем

$$
\operatorname{deg}_{\bar{z}} \mathcal{L}_{1}\left(q_{i}^{1}\right) \leqslant(m+n) N .
$$

С другой стороны, если $p \neq 0$, мы получаем

$$
\operatorname{deg}_{\bar{z}}\left(p q_{2 N-i}^{2}\right) \geqslant \operatorname{deg}_{\bar{z}} q_{2 N-i}^{2}=(m+n) N+2 N-i>(m+n) N .
$$

Противоречие показывает, что $p=0$, и, следовательно, многочлены $q_{i}^{1}, q_{i}^{2}$ являются $m$-гармоническими. Теорема доказана.

СлЕДСтвиЕ 1. Квазиинварианты $q_{i}^{1}, q_{i}^{2}$ вида (9) определены однозначно.

Действительно, линейное пространство $m$-гармоник определено однозначно (см. [9]). Пространство $H_{i}$ однородных $m$-гармоник степени $(m+n) N+i$ двумерно. Следовательно, квазиинварианты $q_{i}^{1}, q_{i}^{2}$ однозначно характеризуются как однородные $m$-гармонические многочлены вида

$$
z^{(m+n) N+i}+O(z \bar{z}), \quad \bar{z}^{(m+n) N+i}+O(z \bar{z})
$$

соответственно, где $O(z \bar{z})$ обозначает полиномы, делящиеся на $z \bar{z}$. 
Наконец, представим явные детерминантные формулы для квазиинвариантов $q_{i}^{1}, q_{i}^{2}$. Введем следующую $(m+n+1) \times(m+n+1)$-матрицу $A=A(i)$ :

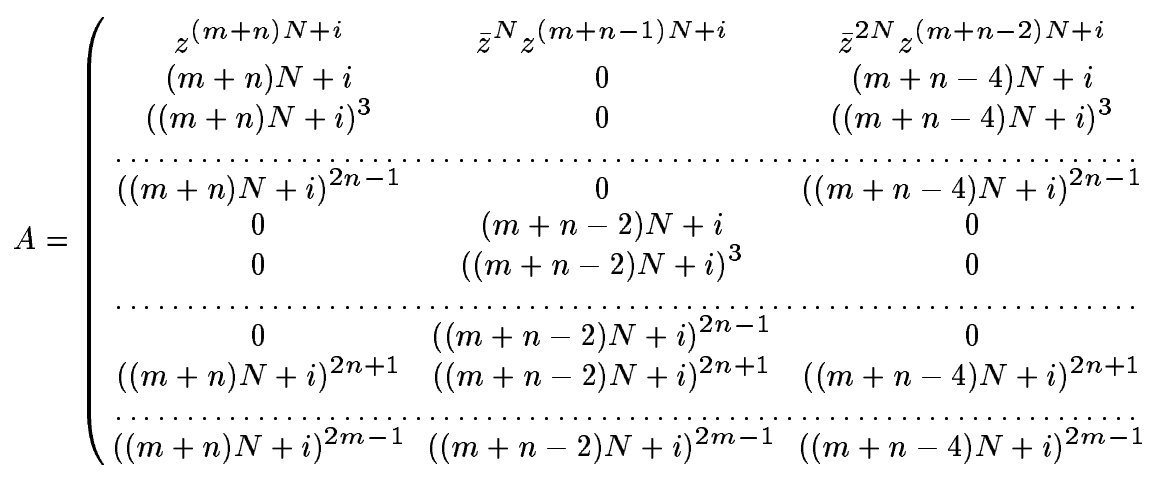

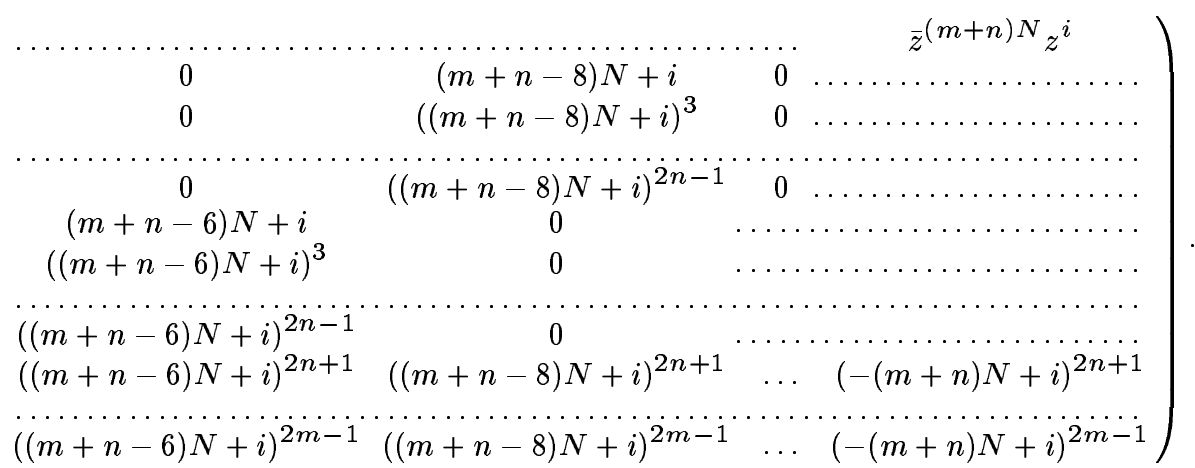

Если мы обозначим $b_{t}=(m+n-2(t-1)) N+i, t=1, \ldots, m+n+1$, то числовые коэффициенты матрицы $A=\left(d_{s t}\right)$ определяются следующим образом. При $s=2, \ldots, n+1$ $d_{s t}=b_{t}^{2 s-3}$, если $t$ нечетно и $d_{s t}=0$ иначе. При $s=n+2, \ldots, 2 n+1 d_{s t}=b_{t}^{2(s-n)-3}$, если $t$ четно и $d_{s t}=0$ иначе. При $s=2 n+2, \ldots, m+n+1 d_{s t}=b_{t}^{2(s-n)-3}$.

Теорема 4. Образуюшие $q_{i}^{1}, q_{i}^{2}, 1 \leqslant i \leqslant 2 N-1, i \neq N$, определяются следующими формулами:

$$
q_{i}^{1}=c \operatorname{det} A, \quad q_{i}^{2}=c \operatorname{det} \bar{A}
$$

где $c=\left(\operatorname{det} A_{1}\right)^{-1}$, а матрица $A_{1}$ получается из $A$ удалением первого столбиа $и$ первой строки.

ДокАЗАТЕльство. Вначале покажем, что $\operatorname{det} A_{1} \neq 0$. При доказательстве предложения 2 мы показали (см. формулы $(11),(12))$, что уравнения квазиинвариантности на коэффициенты многочлена

$$
q_{i}^{1}=\sum_{s=0}^{m+n} a_{s} z^{(m+n-s) N+i} \bar{z}^{N s}, \quad a_{0}=1
$$


имеют вид

$$
\begin{aligned}
& \sum_{\substack{0 \leqslant s \leqslant m+n \\
s=2 k}} a_{s}((m+n-2 s) N+i)^{2 t-1}=0, \quad 1 \leqslant t \leqslant n, \\
& \sum_{\substack{0 \leqslant s \leqslant m+n \\
s=2 k+1}} a_{s}((m+n-2 s) N+i)^{2 t-1}=0, \quad 1 \leqslant t \leqslant n, \\
& \sum_{s=0}^{m+n} a_{s}((m+n-2 s) N+i)^{2 t-1}=0, \quad n<t \leqslant m .
\end{aligned}
$$

По предложению 2 мы знаем, что эта система имеет решение. Условие $\operatorname{det} A_{1} \neq 0$ эквивалентно утверждению, что система (16) имеет единственное решение, так как матрища этой линейной системы есть в точности $A_{1}$. Но решение единственно в соответствии со следствием 1.

Для доказательства теоремы мы должны показать, что

$$
(-1)^{k+1} \frac{\operatorname{det} A_{k}}{\operatorname{det} A_{1}}=a_{k-1}, \quad k=1, \ldots, m+n+1,
$$

где матрища $A_{k}$ получается из $A$ удалением $k$-го столбца и первой строки. По правилу Крамера решение $a_{1}, \ldots, a_{m+n}$ линейной системы (16) задается формулами

$$
a_{k-1}=\frac{\operatorname{det} \widehat{A}_{k}}{\operatorname{det} A_{1}}
$$

где $\widehat{A}_{k}$ есть матрица $A$ с опущенными первым столбцом и первой строкой, в которой вместо $k$-го столбца записан первый столбец с обратным зна́ком. Меняя знак этого столбца и переставляя его на первое место, получаем

$$
\operatorname{det} \widehat{A}_{k}=(-1)(-1)^{k-2} \operatorname{det} A_{k} ;
$$

таким образом, (17) вьполняется. Теорема доказана.

ЗАмЕчАнИЕ. Мы вывели формулы для $q_{i}^{1,2}$ в предположении, что $n<m$. Тем не менее, теорема 4 остается верной, и она дает формулы для базиса в квазиинвариантном модуле, состоящем из $m$-гармонических многочленов, также и в случае $m=n$. Нетрудно увидеть, что в этом случае формулы (15) редуцируются к формулам для $m$-гармонического базиса, полученным в [5].

\section{5. Заключительные замечания.}

5.1. Образуюшие для обших кокстеровских систем. В этой работе завершено описание квазиинвариантного модуля для двумерных кокстеровских систем. Описание образующих $Q^{\mathcal{R}}$ над $S^{G}$ в размерности большей, чем два, неизвестно. В общем случае выбор $m$-гармонических многочленов в качестве базиса невозможен, как это показано Этингофом и Гинзбургом [10]. Однако контрпример, найденный в [10], вьплядит исключительным, так как состоит из системы Кокстера $C_{6}$ с нулевьми кратностями на длинных корнях. Кольцо соответствующих квазиинвариантов может быть описано как модуль над инвариантами группы Кокстера $A_{1}^{6}$ с образующими, которые являются $m$-гармоническими полиномами, связанными с системой $A_{1}^{6}$. Вопрос, когда $m$-гармонические полиномы могут быть выбраны как образующие $Q^{\mathcal{R}}$ над $S^{G}$, является открытым вопросом в размерности, большей 2 . 
5.2. Деформированные системы. В работах [14], [15] исследовались квантовые системы типа Калоджеро-Мозера, связанные с некокстеровскими конфигурациями $\mathcal{A}=$ $\mathcal{A}_{n}(m), \mathcal{C}_{n+1}(m, l)$. Соответствующий оператор второго порядка имеет вид

$$
\mathcal{L}^{\mathcal{A}}=\Delta-\sum_{\alpha \in \mathcal{A}} \frac{2 m_{\alpha}}{(\alpha, x)} \partial_{\alpha}
$$

Как и в кокстеровском случае, коммутативное кольцо квантовых интегралов для $\mathcal{L}^{\mathcal{A}}$ изоморфно кольцу $Q^{\mathcal{A}}$ квазиинвариантов, связанных с системой $\mathcal{A}$. В работе [14] показано, что кольца $Q^{\mathcal{A}}$ являются коэн-маколевьпи, т.е. они свободно порождены над полиномиальными подкольцами $S^{\mathcal{A}}$, которые являются аналогами колец инвариантов в случае систем Кокстера. Вопрос описания колец $Q^{\mathcal{A}}$ и, в частности, вопрос об образующих колец $Q^{\mathcal{A}}$ как модуля над $S^{\mathcal{A}}$ является открытой задачей. Естественное обобщение $m$-гармонических полиномов на некокстеровский случай не дает базис уже в простейшем случае системы $\mathcal{A}_{2}(2)$ (см. [14]).

Благодарности. Я благодарен А. П. Веселову за полезные и стимулирующие обсуждения и Н. Андрееву за помощь.

\section{СПИСОК ЦИТИРОВАННОЙ ЛИТЕРАТУРЫ}

[1] Chalykh O. A., Veselov A.P. Commutative rings of partial differential operators and Lie algebras // Comm. Math. Phys. 1990. V. 126. P. 597-611.

[2] Calogero F. Solution of the one-dimensional $n$-body problem with quadratic and/or inversely quadratic pair potential // J. Math. Phys. 1971. V. 12. P. 419-436.

[3] Moser J. Three integrable hamiltonian systems connected with isospectral deformations // Adv. Math. 1975. V. 16. P. 197-220.

[4] Olshanetsky M. A., Perelomov A. M. Quantum integrable systems related to Lie algebras // Phys. Rep. 1983. V. 94. P. 313-404.

[5] Feigin M., Veselov A.P. Quasi-invariants of Coxeter groups and $m$-harmonic polynomials // Intern. Math. Res. Notices. 2002. V. 10. P. 521-545.

[6] Heckman G. J., Opdam E. M. Root systems and hypergeometric functions I // Compositio Math. 1987. V. 64. P. 329-352.

[7] Taniguchi K. On the symmetry of commuting differential operators with singularities along hyperplanes // E-print math-ph/0309011, 2003.

[8] Волченко К., Козачко А., Мишачев К. Кольцо квазиинвариантов для групп диэдра // Вестн. МГУ. Сер. 1. Матем., мех. 1999. № 1. С. 48-51.

[9] Feigin M., Veselov A.P. Quasi-invariants of Coxeter groups and $m$-harmonic polynomials // E-print math-ph/0105014. 2001.

[10] Etingof P., Ginzburg V. On $m$-quasi-invariants of a Coxeter group // Mosc. Math. J. 2002. V. 2. №3. P. 555-566.

[11] Felder G., Veselov A.P. Action of Coxeter groups on $m$-harmonic polynomials and and Knizhnik-Zamolodchikov equations // Mosc. Math. J. 2003. V. 3. №4. P. 1269-1291.

[12] Berest Yu., Etingof P., Ginzburg V. Cherednik algebras and differential operators on quasi-invariants // E-print math. QA/0111005. 2001.

[13] Feigin M. Rings of Quantum Integrals for Generalized Calogero-Moser Problems. Ph.D. Thesis. UK: Loughborough University, 2003.

[14] Feigin M., Veselov A.P. Quasi-invariants and quantum integrals of the deformed Calogero-Moser systems // Intern. Math. Res. Notices. 2003. V. 46. P. 2487-2511.

[15] Chalykh O.A., Feigin M.V., Veselov A. P. Multidimensional Baker-Akhiezer functions and Huygens' principle // Comm. Math. Phys. 1999. V. 206. P. 533-566. 